\title{
EVALUACIÓN DE RIESGOS DE INCENDIOS EN LOS CORDONES MONTAÑOSOS COSTEROS DE CHILE CENTRAL
}

\author{
Victor QUINTANILLA \\ Departamento de Ingeniería Geográfica \\ Universidad de Santiago de Chile
}

\begin{abstract}
Resúmen: Se analiza el efecto de los incendios de verano sobre la vegetación arbórea y arbustiva de Chile Central lo cual es un fenómeno común en la vegetación de esta zona mediterránea, por lo menos en estos últimos 30 años. Se presenta un caso de estudio en quebradas costeras de la Va Región próximas a las ciudades de Valparaíso y Viña del Mar. Actualmente se reconocen cuatro agrupaciones vegetales dominantes que representan restos de una antigua vegetación más húmeda que existió varios decenios atrás. Destacan las comunidades del bosque esclerófilo costero de Cryptocaria alba y Peunus boldus, el matorral esclerófilo de Quillaja saponaria-Lithrea caústica, el bosque de la "palma chilena"(Jubaea chilensis) y el matorral semixérico abierto de Puya coerulea-Trevoa trinervis. Las comunidades de Lithrea caística se demuestran como plantas que regeneran bien con el fuego, en tanto que Tubaea chilensis y Pulya coerulea se comportan como especies resistentes a éste y no así el matorral de Quillaja saponaria, quien desarrolla una recuperación muy lenta. Al quemarse recurrentemente el estrato subarbustivo y herbáceo, en las fuertes pendientes de las laderas se van dando condiciones para procesos erosivos. Los trabajos de campo han sido apoyados con la aplicación de productos de teledetección, para ir llevando a cabo una cartografía diacrónica de la vegetación (Proyecto FONDECYT N"1950313).
\end{abstract}

Palabras claves: Bosque esclerófilo, fuego, cartografía, palma chilena.

Abstract.: The effect of forestry wildfires on scrub and sclerophyllous vegetation in the mediterranean climate zone of Chile is studied,evaluating both the impact and recovering capacity. The study was carried out in the coastal ravines near Valparaíso-Viña del Mar cities. In the control area a phytogeographical study with the land survey and aerial photographic analysis wiyh 30 years covering. The main conclusions are: today four communities with different strategies of resistance to fire detected: the sclerophyllous forest to Cryptocaria alba-Peumus boldus and the Jubaea chilensis("palma chilena") forest; the scrub of the Quillaja saponaria-Lithrea coustica with vegetative sprout and long youthful phase and the semixeric scrubs to Puya coerulea-Trevon trinervis. Lithrea caustica arboreus species that recuperates best after fire. Quillaja saponaria, shows less recuperation owing to the effects of herbivory uppon its renewalls. Non afected by the fire Jubaen chilensis and Pluy coernlea. The plant regeneration rate shrub and herbaceous stratum was lower than expected and the serious erosion processous we are detected. Jubaea chilensis istin this moment only in dangerous regeneration, by the sever slope the ravines. A important series of diacronic vegetation maps consists of the 1:50.000 sheets 
originally prepared, and information abouth the use of remote-sensing techniques to widland fire mangements is working

Keys words: fire, sclerophyllous forest, maps, chilean palm.

\section{INTRODUCCIÓN}

Los incendios de vegetación en Chile Central $\left(32^{\circ}-35^{\circ}\right.$ Sur) son frecuentes y en ocasiones desembocan en verdaderos desastres. Las características de un clima mediterráneo con un largo y caluroso período seco de hasta 6 meses de duración, otorgan a la vegetación un alto grado de combustibilidad. El área está inserta en la zona de mayor densidad de población del país, lo cual es un factor gravitante en la causa y frecuencia de los fuegos atendiendo que todos ellos son de origen antrópico. Estos siniestros se registran regularmente, en nuestra zona de estudio $\left(32^{\circ}-33^{\circ}\right.$ Sur $)$, desde el inicio de la década del 60 . Este impacto ambiental hoy día se ha transformado en $\mathbf{m}$ problema permanente para las autoridades y la comunidad chilena. Los estudios sobre este tema en el país aún no son suficientes y es por ello, que consideramos que la cartografía de formaciones vegetales combustibles y las cartas de síntesis constituyen un complemento de primer orden para un mejor conocimiento del terreno y de las degradaciones de la vegetación esclerófila de la región.

\section{LOS ANTECEDENTES ECOLÓgICOS DE UN ÁREA DEL CORDÓN COSTERO DE CHILE CENTRAL}

El área montañosa costera regularmente afectada por incendios forestales en 1 a llamada Va Región de Chile', se localiza a espaldas de la conurbación de Valparaíso-Viña del Mar-Quilpué en un relieve constituído por una franja de colinas bajas cuyas alturas máximas no superan los $800 \mathrm{~m}$, con laderas convexas o convexocóncavas disectadas por quebradas (Fig. 1). En contacto con la planicie costera unos 20 3 kilómetros hacia el interior del litoral,se distinguen formas llanas, las cuales se internan entre quebradas. Estas llanuras se interpretan como antiguos niveles de abrasión marina que posteriormente han sido modeladas por procesos erosivos y que han dejado llanos remanentes en las partes altas de los lomajes y fuertes pendientes en los márgenes de los cursos de agua principales.

\footnotetext{
1 En Chile la división administrativa del país se divide en 12 regiones,numeradas correlativamente del I al XII y la Región Metroplitana de Santiago. Cada Región se divide en provincias.
} 
Los suelos corresponden en su mayor parte a la roca granítica descompuesta in situ (maicillo), en la cual es posible reconocer relictos estructurales originales, tales como fracturas, cristales y filones. Estos suelos poseen una alta erosionabilidad frente al escurrimiento de aguas de lluvias y a menudo por ello, aflora en superficie un material arcilloso.

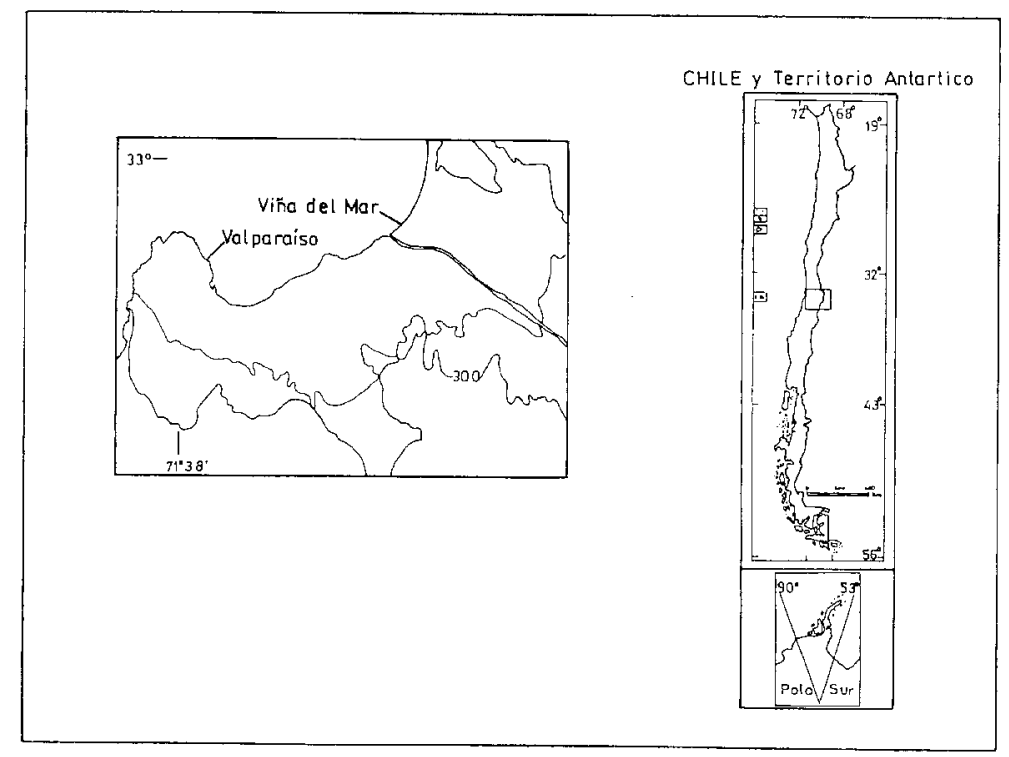

Figura 1.- Area de estudio.

Las colinas costeras forman una unidad orográfica muy influenciada por 1 a proximidad del mar desde donde las neblinas superficiales se desplazan hacia la diversidad de quebradas existentes, contribuyendo a presentarse un clima local tipicamente templado con respecto al de tendencia de carácter mediterráneo que predomina en gran parte de la región con un monto anual de precipitaciones en la costa del orden de los $400 \mathrm{~mm}$ (QUINTANILLA, 1975). Es por estas condiciones que en el área de estudio (que cubre aproximadamente unas 6.000 hás.) se presentan diferencias significativas en las condiciones de humedad entre las laderas de exposición sur y las expuestas al norte. Esta condición determina la existencia de tipos de vegetación diferentes en las quebradas y fondos de valles.

Estas unidades vegetales se clasifican en 6 agrupaciones predominantes (VILLASEÑOR, 1977) y corresponden en principalmente, a comunidades testigos de la cubierta vegetal primitiva que cubrió siglos atrás la zona. La búsqueda de leña, 
madera y frutos junto con los efectos del fuego, son los principales agentes responsables del estado actual de la cobertura vegetal : a) Bosque esclerófilo costero que crece a 1 oeste de la cordillera costera por encima de los $200 \mathrm{~m}$, el cual, según las cantidad de agua disponible en el medio, posee varios exponentes. b) Bosque higrófito formado por árboles latifoliados que crecen a orillas de agua o en lugares de alta humedad, en laderas de umbría y fondos de quebradas. c) Matorral esclerófilo, formación arbustiva que posee coberturas variables producto de la tala y quema de las unidades anteriores. d) Matorral higrófito constituído por la degradación del bosque higrófilo y se sitúa a orillas de agua en esteros y canales. e) Matorral xerófilo en laderas de exposición norte y que crecen como comunidades secundarias. f) Palmares, comunidades de palma chilena situadas preferentemente en partes bajas de quebradas en exposición al mar. g) Puyales, agrupaciones en que dominan las especies del género Puya con una forma de crecimiento única. Las comunidades degradadas han desarrollado una adaptación morfológica y fisiológica a la sequedad, lo cual les otorga un riesgo importante de inflamabilidad.

\section{LOS RIESGOS RECURRENTES DE INCENDIOS EN LA ZONA COSTERA DE CHILE CENTRAL (Va REGION)}

Los fuegos en los montes de Chile se remontan desde la época colonial y como no existen en el país ecosistemas pirófitos, el origen de ellos se debe a las actividades antrópicas siendo la mayoría de los incendios de tipo superficial. Cartwright (DONOSO, 1981) estima que en los 50 años anteriores a 1968 se habían quemado en Chile alrededor de 1.200 .000 hectáreas de bosques, equivalentes a 4 mil millones de pulgadas de madera.

Desde fines de la década de los 60, el incremento de los siniestros en la temporada del fuego en el país (noviembre a abril del año siguiente) ha ido en $\mathbf{n}$ notorio incremento tanto en las formaciones nativas como en plantaciones forestales (Fig. 2). En un análisis de la superficie quemada y del número de incendios por regiones en Chile, hecho por SÁIZ (1990), se observa para un período de 10 temporadas una distribución irregular con una mayor concentración en las regiones Va $\left(32^{\circ} \mathrm{S}\right.$ aproximadamente) y Villava $\left(37^{\circ}-38^{\circ} \mathrm{S}\right)$, y con una menor intensidad en las regiones Vila (36 S), Metropolitana $\left(33^{\circ}-34^{\circ}\right.$ S), IXa y Xa $\left(39^{\circ}-40^{\circ}\right.$ S) (Fig. 3). Esta distribución coincide con la mayor concentración de población, la importante presencia de vegetación nativa, y de plantaciones exóticas de pinos y eucaliptos.

La causalidad de estos fuegos es debida exclusivamente a actividades humanas como: faenas agropecuarias, faenas forestales, actividades de recreación y deportivas, tránsito y transporte, obras viales, etc. 


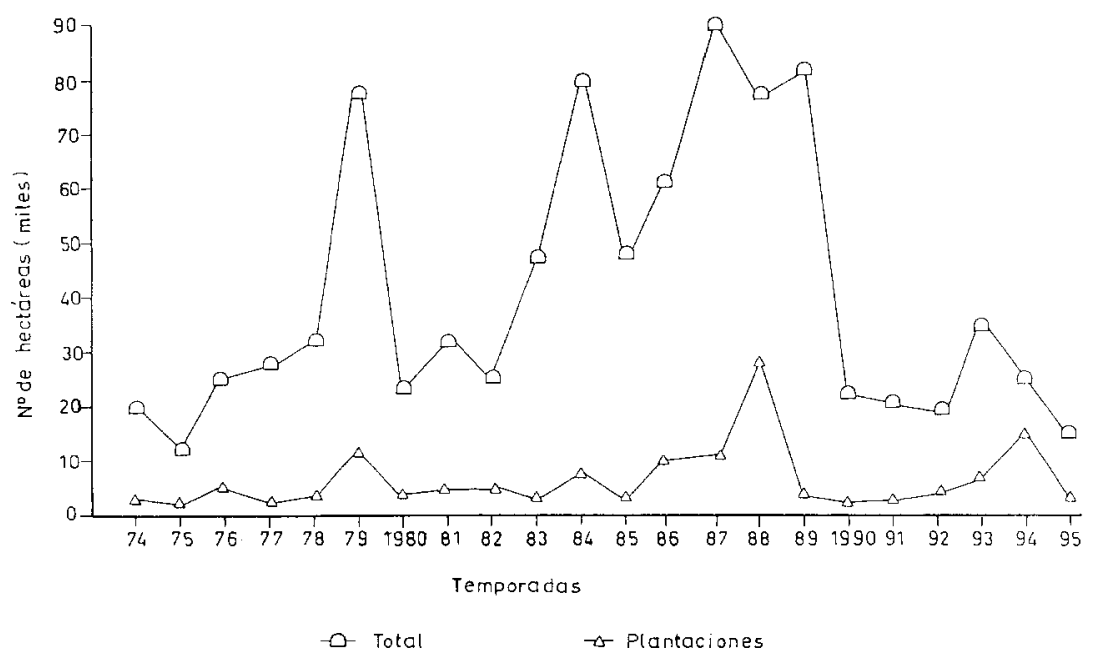

Figura 2.- Evolución del $\mathrm{n}^{\circ}$ de hectáreas quemadas y relación entre vegetación nativa y plantaciones en Chile (1974-1995).

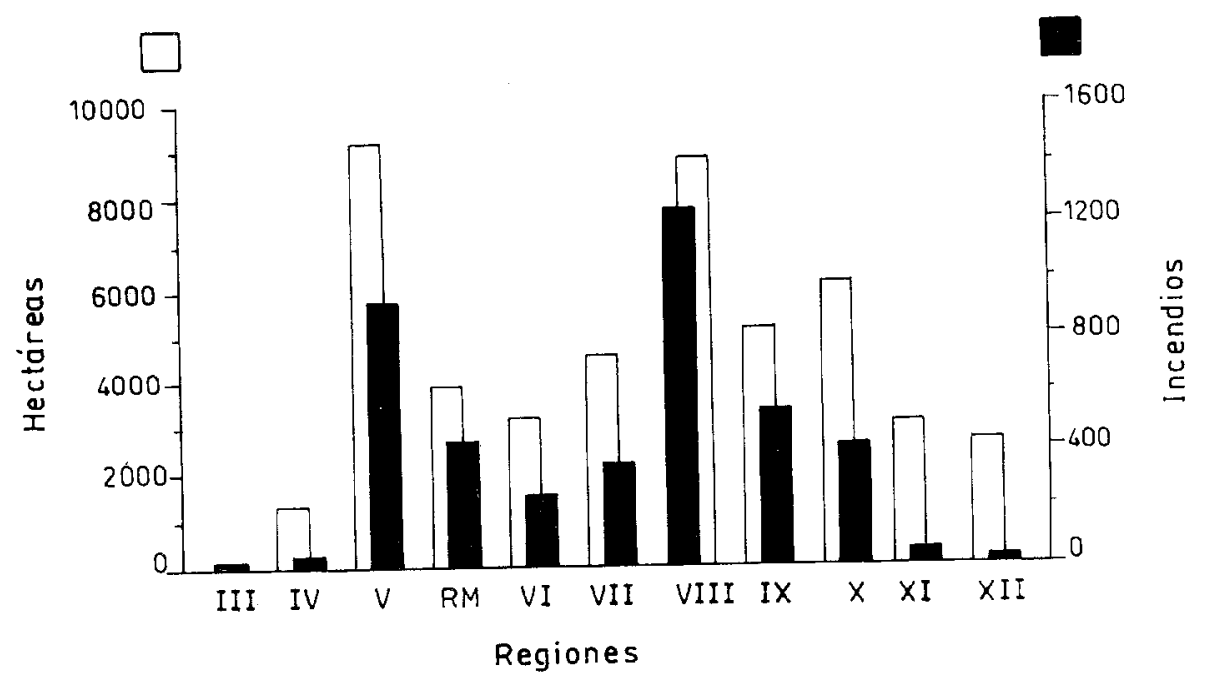

Figura 3.- Distribución por regiones del $\mathrm{n}^{\circ}$ de incendios y hectáreas quemadas de 1978-79 a 1989-90 (Saiz, 1990). 
La Va Región concentra una alta densidad de población urbana (92\%) y se localiza preferentemente en el borde costero y en las colinas de las terrazas marinas, como también en las riberas de los principales cursos fluviales. Por ello, esta masa poblacional constituye un alto riesgo potencial de incendios en las áreas silvestres del entorno cercano a estos habitats.

Analizando, según SÁIZ (op.cit.), el total de hectáreas quemadas desde 1 a perspectiva del tipo de combustible comprometido, (Fig. 4), se constató que el $94 \%$ correspondió a vegetación nativa con distribución casi similar entre bosques, matorrales y pastizales. En la Va Región la distribución espacial de estas variables es irregular concentrándose en la periferia suburbana de las localidades de Quillota, San Antonio, Valparaíso y Viña del Mar el $99 \%$ de los incendios estudiados, para un período de 10 temporadas (Fig. 5).

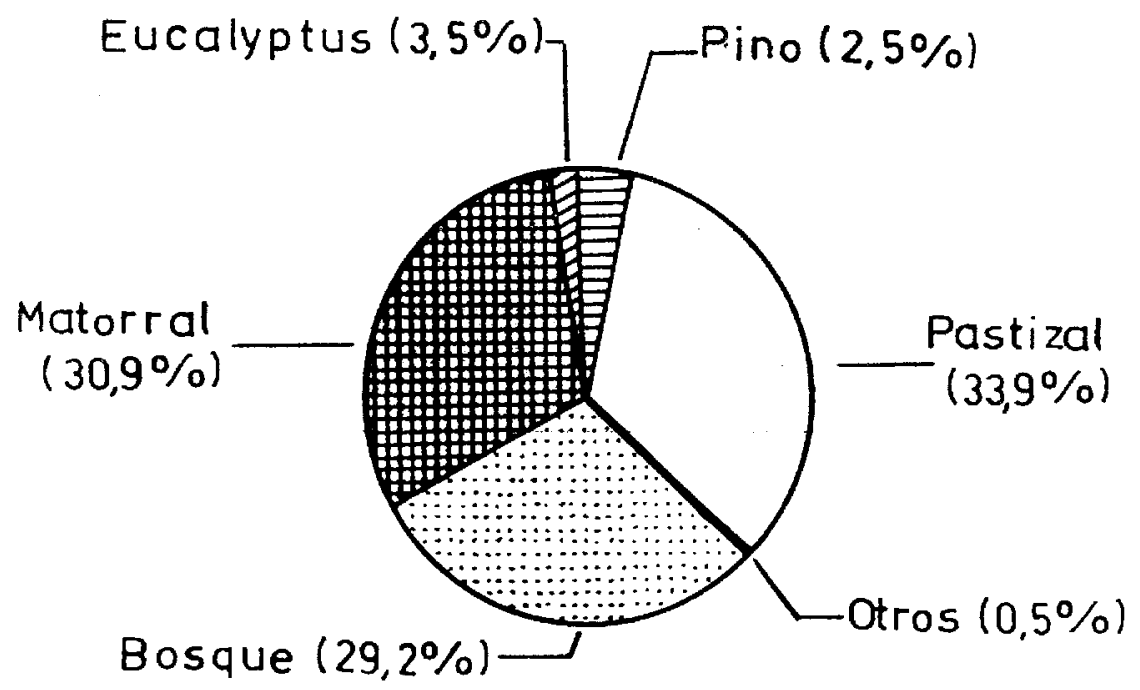

Figura 4.- Distribución de los tipos de combustible forestal en la V Región. Temporadas 1990-1995. 


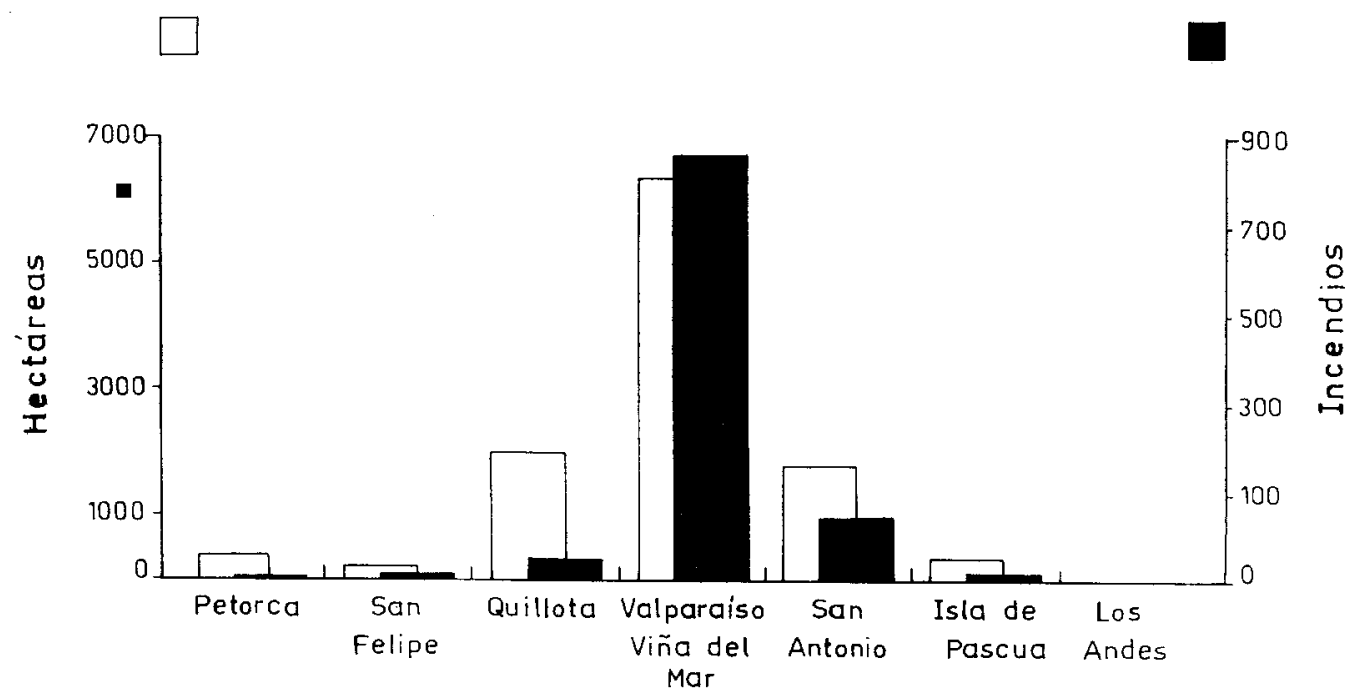

Figura 5.- Promedio de incendios y hectáreas quemadas por provincias en la $\mathrm{V}$ región de 1978-79 a 1989-90.

\section{LA VULNERABILIDAD AL FUEGO DE LA VEGETACIÓN DEL CORDÓN COSTERO}

- Objetivos y metodología

A pesar que en Chile es decisivo el elemento humano en el surgimiento de los incendios, coadyudado por las condiciones meteorológicas locales, en este fenómeno no debe restárse importancia a las características propias de la vegetación. Efectivamente entre los factores externos (pendientes, vientos, etc.) que influencian el comportamiento del fuego, los vegetales son el factor esencial de los incendios por cuanto las plantas constituyen el elemento combustible que se quema. Por tanto 1 a cartografía de la vegetación orientada hacia el tema de los combustibles vegetales, es una etapa preliminar de la síntesis cartográfica del riesgo de incendio (TRABAUD 1970 y 1992). 
- La cartografía de las formaciones vegetales combustibles

Tradicionalmente el mapeo de la cubierta vegetal entrega una visión potencial del recurso o también, una visión gráfica del momento actual de los tipos de comunidades de plantas que están presentes en un espacio dado, aportando pocos antecedentes para la apreciación del combustible vegetal existente. Sin embargo para esta zona se han llevado acabo algunos esfuerzos pioneros en mapeos de incendios (ZUNINO y RIVEROS ,1990). Nuevos antecedentes se procuran incorporar a través de la carta de formaciones vegetales combustibles donde se integran variables diversificadas y representativas que debieran, por una parte, facilitar el conocimiento de los combustibles vegetales y por otro, guiarnos en el conocimiento de los factores que determinan la naturaleza misma de esas formaciones. Integrando las variables propias de los combustibles, este tipo de cartografía traduce de modo objetivo la complejidad natural de la vegetación (Fig. 6). Según TRABAUD (1992-93), la ejecución de la carta pasa por dos etapas:

a) Determinación de un conjunto de parcelas isófenas: Además de los insustituibles muestreos de campo, se lleva a cabo un trabajo preliminar consistente en una interpretación de diferentes fuentes de información (fotos aéreas, cartas de uso del suelo, etc.), lo que permite, por intermedio de una gran zonación a gran escala, destacar las áreas en las cuales la vegetación muestra, tanto desde el punto de vista de la estructura como de la composición florística, una cierta homogeneidad.

Ajustando en el estudio de campo las áreas previamente establecidas, se continúa en la segunda etapa para un análisis más detallado de la vegetación por cada parcela isófena.

b) Identificación de las comunidades vegetales. Ellas se identifican a través de diversos elementos: estructura, talla, caducidad o no, porcentaje de recubrimiento, etc. También se deben considerar otros elementos importantes; por ejemplo, la naturaleza del tipo de agrupación: se refiere a la distribución horizontal y vertical de los individuos vegetales y de sus órganos vegetativos. Disposición de las parcelas en estratos Herbáceos (H): 0.10 a 0,50 cm; en Leñosos Bajos (LB) de 0,50 a $200 \mathrm{~cm}$; y Leñosos Altos (LA), estrato superior a $200 \mathrm{~cm}$. Entre estas categorías se pueden diferenciar a su vez numerosos rangos. Así por ejemplo, nosotros individualizamos 8 unidades de estratificación fundamentales como se observa en la Figura 6.

c) Tipos de combustible y de las especies vegetales dominantes: se refiere a 1 a influencia que poseen las plantas sobre el estado de inflamabilidad y de combustibilidad de la agrupación. En el caso de nuestro trabajo se aplica sobre todo a las características de la vegetación del matorral que es el tipo de vegetación predominante en la actualidad. 


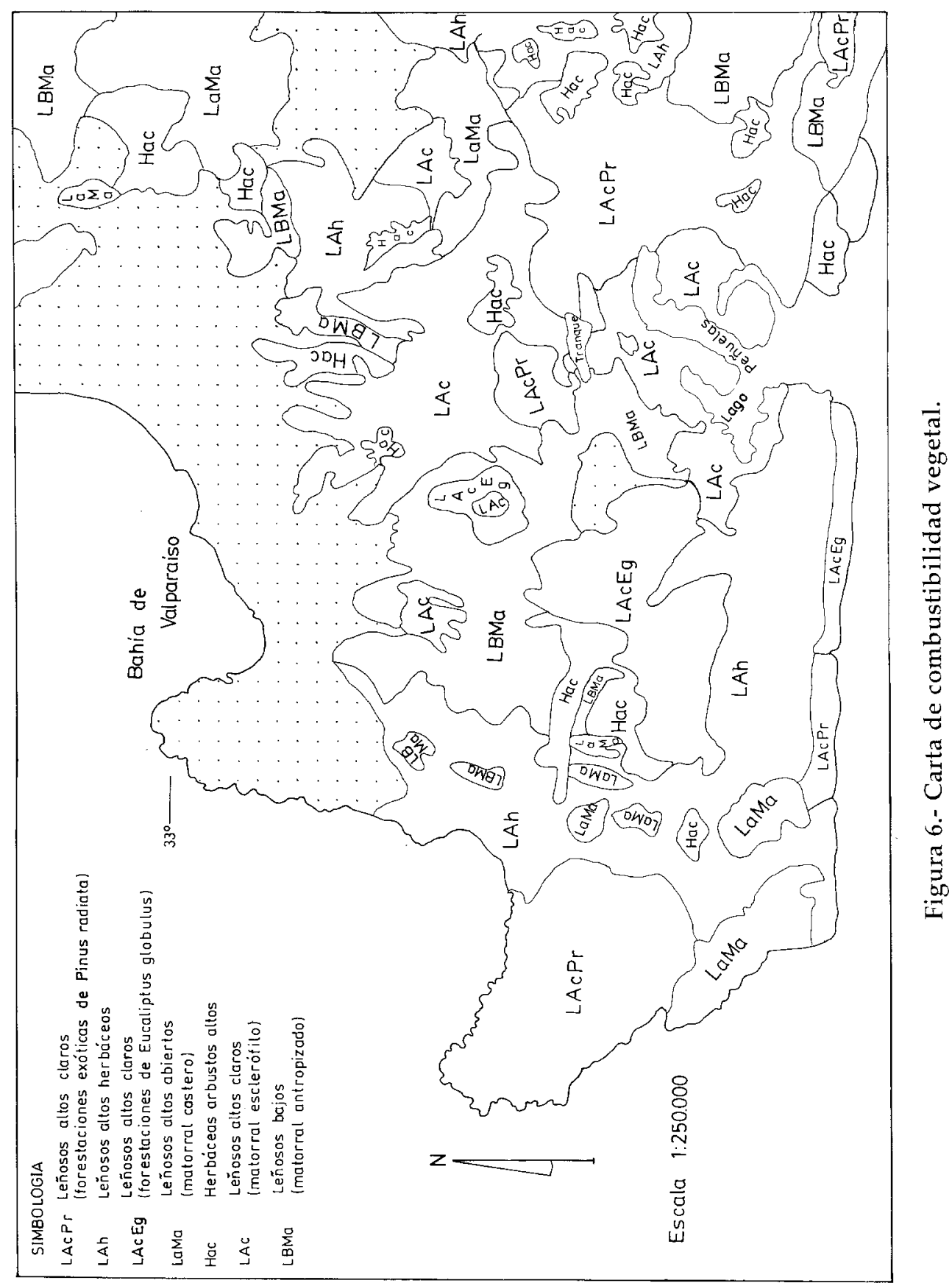


Observamos por ejemplo que el matorral suele ser una formación densa en la que a la proximidad de la copa de los arbustos vecinos se suma el estrato herbáceo, el cual se seca tempranamente en el verano para conformar factores que apoyan el rápido avance del fuego una vez iniciado. Otro factor que favorece el avance del fuego en plantas de matorral es que las hojas se mantienen activas por períodos de uno o dos años, excepcionalmente tres o cuatro, lo que forma un permanente estrato de hojas caídas en distintos grados de descomposición. A esto se suma la existencia de plantas que botan ramas completas, trozos de corteza y otros elementos cuyo conjunto constituye un excelente material susceptible de ser quemado.

Estos factores se ven incrementados por la presencia de otras características que hacen muy combustible la vegetación. Así por ejemplo la presencia de resinas, aceites esenciales, pelos, espinas, un bajo contenido de agua y las gruesas cutículas presentes en las hojas de las plantas dominantes favorecen la combustión (AVILA y ot., 1988).

En el campo habría que pronunciarse por identificar 2 ó 3 especies (sobre todo en las comunidades de matorrales y malezas) para graficar en la carta las más dominantes, afectándoles en la simbología su nombre científico mediante iniciales con letras minúsculas. Por ejemplo: LAa.Lc $=$ Leñosos altos abiertos con dominancia de Lithrea caística. Hacer referencia a un número mayor de plantas presentes hace muy confuso el uso del mapa, no obstante que todos los individuos desempeñan un rol importante con respecto a la vulnerabilidad y sensibilidad al fuego.

La metodología propuesta por Trabaud también incluye la denominada tasa de recubrimiento de los diferentes estratos constitutivos, la cual es igual a la suma de la proyección vertical de un estrato dado con relación al área total de muestro, correspondiente al biovolumen que representa el volumen total ocupado por el material vegetal de una formación susceptible de quemarse después de un incendio (TRABAUD, 1992-93). En esta fase nosotros estamos todavía trabajando y esperamos aportar resultados en otra oportunidad. Del mismo modo sucederá con otro aspecto de esta metodología, la cual considera igualmente normas para enfrentar la lucha contra los incendios. En este aspecto aún en Chile, no hay suficiente planificación y coordinación entre las organizaciones que luchan contra el fuego.

En el mapeo final que hemos adoptado para la descripción de las formaciones vegetales, en la carta proponemos entonces la simbología siguiente: Ej.: Lac: tipo de formación (Leñosos altos claros), $\mathrm{Pr}=$ Pinus radiata, especie dominante (forestaciones) $=\mathrm{LAcPr}$; o Laa $=$ Leñosos altos abiertos; ma= matorral mixto costero: LA.a. m.a., o $\mathrm{Hac}=$ Herbáceas y arbustos claros. A estas letras se pueden agregar una o dos cifras cuando se calcula el índice de biovolumen y luego, números romanos si se mide en los bosques el criterio ADP de los árboles. Al trabajar la cartografía en mediana escala, 
representamos a la formación más predominante en el recubrimiento superficial de las parcelas, cuando están presentes más de una comunidad vegetal.

\section{LOS RIESGOS POTENCIALES DE INCENDIO EN LAS FORMACIONES VEGETALES PRESENTES}

Dado que estas unidades vegetacionales tienen el carácter de conformar un gran arco periférico a la conurbación de Valparaíso-Viña del Mar (800.000 habitantes), de suyo ellas adquieren un rasgo de alto riesgo de incendio casi permanente durante el año. Sin embargo, tales comunidades vegetales combustibles han demostrado que poseen una mayor o menor aptitud de inflamarse bajo el efecto del fuerte y rápido calentamiento del medio local en el cual se desarrollan (sensibilidad al fuego) y no siempre tanto, con respecto a la estructura y composición de sus órganos vegetativos. En el ensayo que previamente hicimos para observar qué relación tenían las formaciones vegetales combustibles con respecto a la sensibilidad al fuego, no encontramos una correspondencia "coherente". Así por ejemplo, las comunidades de Leñosas altas herbáceas (LAh) no corresponden sino a un área de riesgo potencial moderado de incendio; o ciertos sectores con recubrimiento de Leñosos altos claros (LAc) poseen in rango de riesgo débil o bajo (Fig. 7). Observaciones de terreno en fases de pre y post incendio, apoyadas con mediciones en parcelas test, nos entregaron ciertas explicaciones al respecto. Establecimos un índice de sensibilidad muy simple tomando en cuenta la breve experiencia histórica conocida, con respecto a la inflamabilidad de las especies dominantes en las cuales la proporción de formas biológicas y su estructura permiten la propagación del fuego con mayor rapidez ${ }^{2}$.

La mayor o menor presencia de estas variables nos aproximaron a un indice de sensibilidad de riesgo al fuego desde muy elevado a débil y que no es tan riguroso como el propuesto por TRABAUD (1980). Somos conscientes que estas mediciones aún son muy estimativas considerando sobre todo dos antecedentes. El primero es que en Chile no hay plantas pirófitas y el segundo, es que el origen de los siniestros en el país siempre es de responsabilidad humana sea directa o indirecta. En consecuencia cualquier formación vegetal, por muy insensible que pareciera ser al fuego, puede arder totalmente o por mucho tiempo. Por tanto el énfasis para discriminar qué agrupaciones entonces pueden denotar un riesgo muy elevado, moderado o débil al fuego, nosotros por ahora lo hemos puesto en gran parte en cierto modo con respecto a una variable humana.

\footnotetext{
${ }^{2}$ En Chile sólo hay registros de incendios forestales únicamente a partir de alrededor de 1960 y con datos, no siempre confiables.
} 


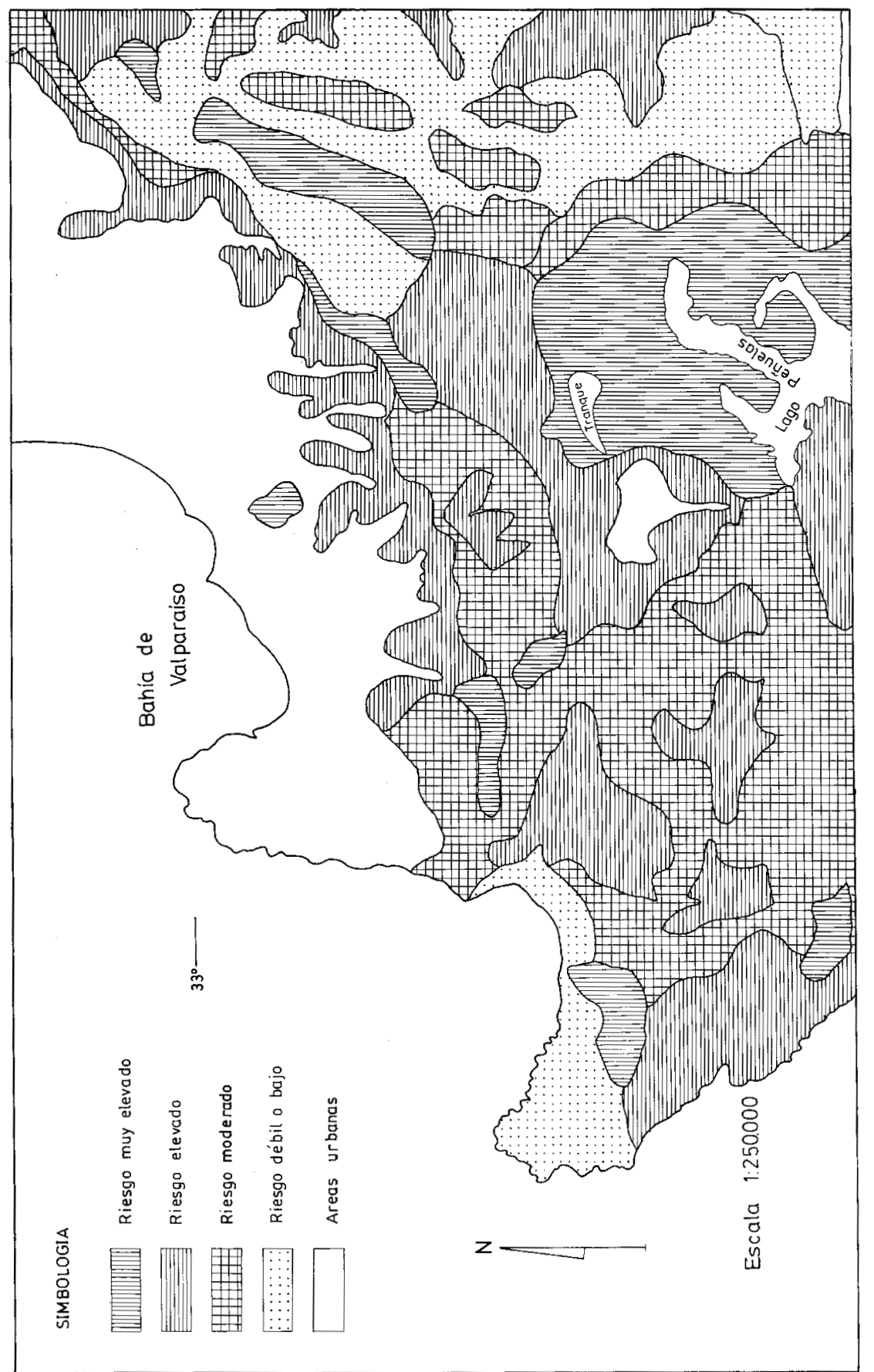




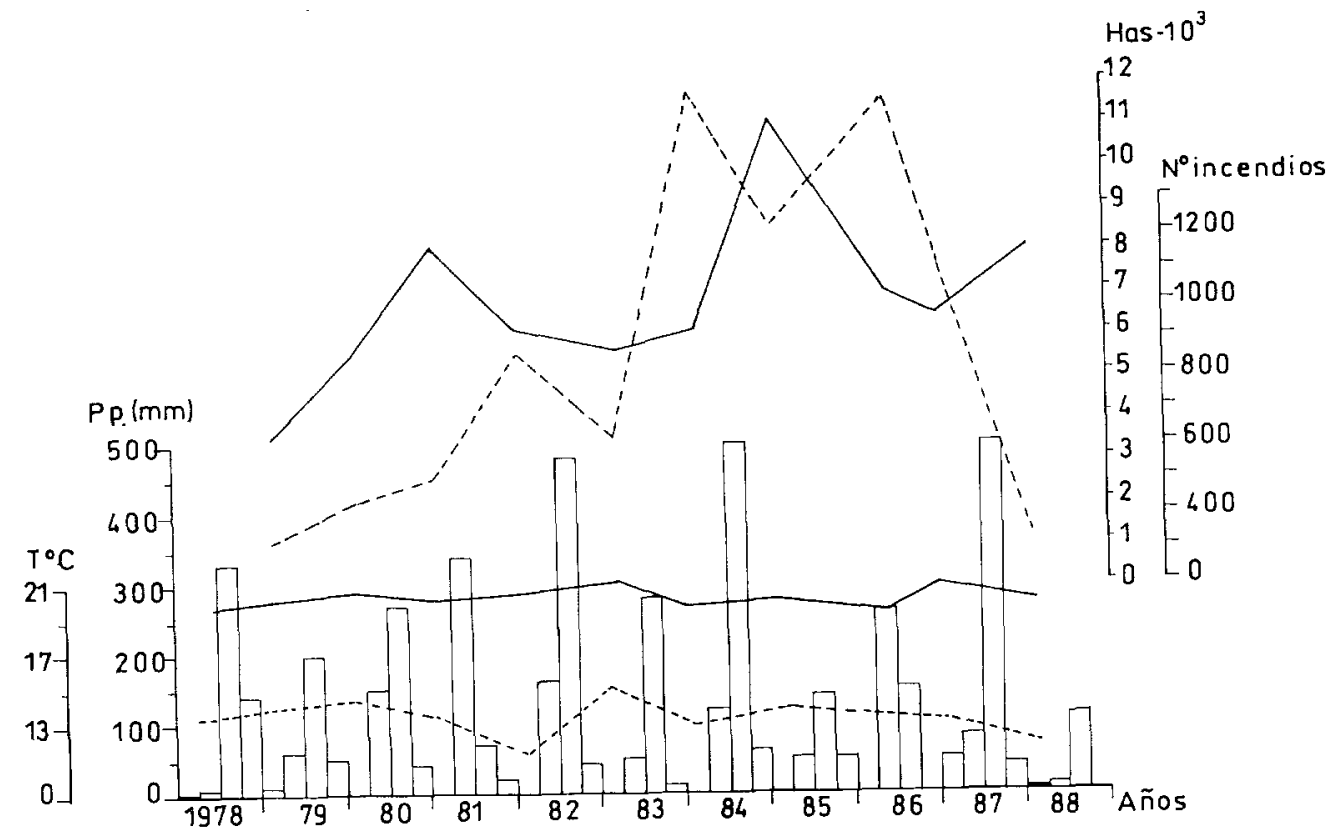

Figura 8.- V región. Relación entre precipitaciones trimestrales (mm), temperaturas medias máximas y medias mínimas del período $\operatorname{seco}\left({ }^{\circ} \mathrm{C}\right), \mathbf{n}^{\circ}$ de incendios y hectáreas quemadas (SÁIZ, 1990).

Considerando lo dicho entonces, poseen un riesgo moderado de incendio aquellas formaciones que están lejos y cuya accesibilidad para combatirlas es muy dificultosa sea por la distancia o por lo sinuoso del acceso y por esto a su vez adquieren un alto grado de inflamabilidad. Por otra parte poseen un riesgo elevado de incendio, también las agrupaciones que están muy próximas de asentamientos humanos. Evidentemente que esto no excluye considerar las características biológicas o de estructura de las plantas como por ejemplo: la caducidad durante el verano, la existencia de especies resinosas, la fuerte deshidratación en el largo y seco verano;si descartar además el rol que desempeñan las variables meteorológicas locales (Fig. 8). 


\section{LA SENSIBILIDAD AL FUEGO DE LAS DIFERENTES COMUNIDADES VEGETALES}

La comparación de una parte de las dos cartas de síntesis anteriormente presentadas, contribuye en parte a caracterizar la respuesta de las diferentes agrupaciones vegetales ante el potente factor de degradación que asume el fuego.

En la zona de estudio- $y$ de acuerdo con VILLASEÑOR 1997-predominan 3 tipos de formaciones vegetales: El Bosque Esclerófilo Costero, el Matorral Esclerófilo y el Matorral Xerófilo. Por su parte, al interior de estas formaciones y según el efecto de las condiciones ecológicas locales, se identifican otras agrupaciones más específicas como las siguientes:

- En el Bosque Esclerófilo Costero de este área se encuentra por ejemplo la agrupación "Peumo-Boldo" que crece en laderas de exposición sur entre los 230 y 300 m, en pendientes de unos $25^{\circ} \mathrm{y}$ de humedad moderada. Dominan especies esclerófilas típicas de Chile central como el "peumo"(Cryptocaria alba), el "boldo"(Peumus boldus), el "corcolén"(Azara celastrina) y el "molle"(Schinus latifolius). En laderas sombrías, la comunidad alcanza un desarrollo importante en sus doseles superiores, 10 cual favorece los incendios de copa en el área. Al sur poniente del Lago Peñuelas, esta agrupación posee actualmente una representación muy reducida precisamente por efecto del fuego.

- Sobre los $300 \mathrm{~m}$, en las colinas costeras y en laderas de exposición oeste y noroeste con baja humedad y en pendientes mayores de $15^{\circ}$, se desarrolla el bosque de"peumo"(Cryptocaria alba) y "quillay"(Quillaja saponaria), el cual suele estar acompañado del "litre" (Lithrea caústica). Es muy variable en cuanto a densidad de su dosel por lo cual a menudo presenta baja cobertura. Sin embargo algunas de su especies arbustivas acompañantes son vulnerables al fuego (Podanthus mitiqui, Trevoa trinervis, Gallium aparine, Colliguaja odorifera,etc.)

- En quebradas con influencia oceánica y sin desplazarse por encima de los $350 \mathrm{~m}$, crece el bosque de palmas. La "palma chilena"(Jubaea chilensis) es la especie dominante y sobresaliente entre matorrales y bosquetes esclerófilos especialmente de Lithrea caústica quien crece en un estrato inferior. En la zona la palma es el único árbol que se demuestra invulnerable al fuego, aunque se queme todo su cortejo florístico acompañante sobre todo si son arbustos. Jubaea chilensis, árbol que puede alcanzar hasta $30 \mathrm{~m}$ de altura ha resistido por muchos años el impacto de los fuegos de la zona.

- El fuego junto con la tala, ha contribuido en alta proporción a la degradación del bosque esclerófilo fundamentalmente de "litre" y "boldo", transformándolo paulatinamente en un matorral de densidad variable y que en algunos lugares alcanza 
el estrato arbóreo. La comunidad se quema con frecuencia en desmedro de Peumuls boldus y no así de Lithrea caústica. Estudios hechos en la región donde después de 2 años de ocurrido un intenso incendio, comprobaron que este árbol adquiere un porcentaje de rebrote de un $70 \%$. Algo similar se ha testificado en las riberas orientales del Lago Peñuelas donde la agrupación se quema con frecuencia.

- Cuando este matorral entra en un evidente proceso de degradación se pierde Peumus boldus y se reduce la presencia de Lithrea caústica en favor de un arbusto invasor como es el "corontillo"-Eringium paniculatum - el cual hemos observado que coloniza rápidamente estos espacios después del paso del fuego. A los 28 días después de un incendio en febrero de 1997, encontramos numerosos renuevos de la especie y particularmente en laderas de exposición sur. Igual fenómeno ocurre en gran parte en el matorral de Peumo-Boldo cuando éste se ha quemado en varias ocasiones, como sucedió 30 años atrás en el área del Lago Peñuelas y antes que llevaran a cabo las forestaciones de Pinus radiata y posteriormente de Eucaliptus globulus.

- En laderas de exposición oceánica y en el fondo de algunas quebradas quedan aún pequeñas comunidades de "maqui" y "lilén" (Aristotelia chilensis y Psoralea glandulosa) quienes conforman restos de la degradación del bosque higrófito de "patagua"(Myrceugenia exsucca) y "canelo"(Drimys winteri). Esta agrupación es poco afectada por el fuego y por el contrario tiende a ocupar terrenos quemados, excepto en el área del Lago Peñuelas donde casi se ha perdido.

- En sectores de poca pendiente y de moderada humedad se ha ido desarrollando el matorral de "quebracho"(Cassia stipulacea), el cual logra sobrevivir mejor al fuego cuando ha superado la talla herbácea. Actualmente, aunque disperso, se localiza en la periferia de las quebradas.

- En numerosas laderas de exposición norte y preferentemente en afloramientos rocosos, se desarrolla una formación xerófita conformada por el "Puyal" de "chagualquisco". Lo caracterizan el "chagual"(Puya chilensis), la "puya"(Puya berteroana) y el "quisco"(Echinopsis chilensis), los cuales pueden encontrarse desde los 150 a los $350 \mathrm{~m}$. Esta comunidad en general demuestra una baja sensibilidad ante el fuego, por cuanto la mayoría de sus componentes pasan casi indemnes la temporada de incendios,excepto el chagual a quien se le quema una parte de su estructura.

- Ocupando lugares húmedos, algunos fondos de quebradas y en laderas con pendientes moderadas, tenemos acá también un matorral de "colihual "o "colihue" (Chusquea cumingii. Corresponde a una degradación del bosque de LitreBoldo, poco o medianamente alterado. Presenta una cobertura alta y puede encontrársele tanto en fondos de quebradas como en laderas de exposición marina. Es una bambúsea perenne de gran enraizamiento con un denso ramaje en cañas simples y 
que después del paso del fuego en el bosque o en el matorral, desarrolla una gran fuerza de colonización adquiriendo el carácter de invasora en los sectores de mayor humedad. E1 "colihue" es un arbusto trepador que alcanza hasta $2,5 \mathrm{~m}$ de altura y de alta combustibilidad, a través de numerosas cañas libres y secas en el verano. Cuando estas comunidades se encuentran en la periferia del bosque o del matorral, el "colihual" arde intensamente llevando las llamas hacia las otras agrupaciones.

Sobre planos altos y áreas más llanas de esta zona de estudio, se han desarrollado en estos últimos años plantaciones de carácter comercial unas, y de protección de riberas de aguas otras. Se localizan fundamentalmente en torno al Lago Peñuelas, Tranque Las Cenizas y la punta Curauma. Corresponden a agrupaciones leñosas altas y claras (LAC) caracterizadas por un sotobosque y estrato arborescente relativamente pobre y abierto. La formación adquiere un alto riesgo para incendios de copa, los cuales a menudo se propagan a las comunidades vecinas seudonativas de matorral.

\section{EL VALOR DEL ANÁLISIS CARTOGRÁFICO. CONCLUSIONES}

La cartografía de formaciones vegetales combustibles y de síntesis de riesgo de fuego que pueden elaborarse son de gran utilidad, especialmente si ellas se levantan a gran escala. Sobre el particular en Chile, este tipo de cartografía está poco utilizada.

Las características de la combustibilidad varían de manera notable en el tiempo y en el espacio (talla, masa, distribución, presión antrópica,etc.) por lo cual se hace necesario (o ideal) realizar frecuentes actualizaciones de la carta. Puede pensarse también que estos mapas son muy empíricos, por ejemplo en cuanto a lo relativo que puede ser individualizar al fuego en lo que denominamos carta de sensibilidad, cuando buscamos los sectores de riesgo de ignición del fuego. Sólo está claro el punto, cuando localizamos el agente iniciador de los incendios con frecuencia ubicados en la proximidad de caminos y de habitaciones.

También la apreciación respecto al comportamiento del fuego en cuanto a la expansión y recubrimiento superficial del incendio, debe considerar tanto variables meteorológicas como los parámetros relativos a la vegetación: especies dominantes, caducidad o no de las plantas, estructura y combustibilidad y otros. Tampoco aquí deben descartarse los factores topográficos.

En todo caso esta representación cartográfica preliminar que proponemos, y que para el caso de Chile estamos en una de sus fases de desarrollo, en vías a un tratamiento de GIS, contituye un instrumento de trabajo fundamental en la 
investigación no sólo con respecto al fuego y la vegetación que constantemente se degrada, sino también para proponer acciones para la detección del fuego y disponer a su vez de conocimientos más precisos de las comunidades de plantas, a fin de poder enfrentar la lucha contra estos siniestros.

Estos antecedentes son indispensables, como afirma TRABAUD (1991-92), para la elaboración de cartas de síntesis de riesgos y sensibilidad al fuego cuyo carácter de documentos gráficos integrados, incorporan la combinación de parámetros básicos como la estructura de las agrupaciones, los valores de pendientes, la circulación del viento, la frecuencia de la acción antrópica,etc. Ellas sobre todo pueden contribuir eficazmente en el dominio de la prevención de incendios.

\section{BIBLIOGRAFIA}

ALJARO, M.E. y ot. (1988): Incendios en la vegetación mediterránea de Chile. En FUENTES, E. y PRENAFETA, S. (Ed.): Ecología del paisaje en Chile Central. 81-87, Vic. Ac. Universidad Católica de Chile.

DONOSO, C.(1981): Ecología Forestal. El bosque y su medio ambiente. Editorial Universitaria. 260 p, Santiago. Chile.

QUINTANILlA, V. (1975): "La carta bioclimática de Chile Central" Rev. Geográfica de Valparaíso, 6, 33-58. Valparaíso. Chile.

QUINTANILLA, V. (1983): Biogeografía de Chile. 230 p, Vol.III. Col. Geografía de Chile. Inst. Geográfico Militar. Santiago.

QUiNTANILLA, V. (1996): "Geocartografía e incendios de vegetación en sectores de Chile central". Rev. Geográfica Terra Australis, 4, 143-157. Santiago.

TRABAUD, L. (1970): "Le comportement du feu dans les incendies de forêt". Rev. Tecnique du Feu, 103, 1-15. Montpellier.

TRABAUD, T. (1980): Impact biologique et écologique des feux de végétation sur l'organisation, la structure et l'évolution de la végétation des zones de garrigues du Bas Languedoc. Thèse d'Etat Sci. nat. USTL. 288 p, Montpellier.

TRABAUD, L. (1992-1993): "Évolution des risques d'incendies dans une zone sensible: les Aspres (Pyrénnees Orientales)". Revute Geogr. des Pyrennées et du Sud-Ouest. 63, 34-51. Toulouse.

SÁIZ , F. (1990): "Incendios forestales en el Parque nacional La Campana. Sector Ocoa, Vta. Región. Chile. Anales Museo Hist. Natural de Vaiparaíso. 21, 5-14. Valparaíso.

VILLASEÑOR, R.y SAIZ, F.(1990): Incendios forestales en el Parque nacional La Campana, Sector Ocoa.Vta. Región. Chile. II: Efectos sobre el estrato Arbustivo-Arbóreo". Anales Muıseo Hist. Natural de Valparaíso. 21, 16-26. Valparaíso. 
VILLASENOR, R. (1997): Unidades de vegetación de los cerros costeros de la provincia de Valparaíso. Chile. 6 p. (inédito).

ZUNINO, S. y RIVEROS, G. (1990): "Cartografía de los incendios forestales de la Vta. Región. Chile" Anales Murseo Hist. Natural de Valparaíso, 21, 89-93. Valparaíso. 